This work is on a Creative Commons Attribution 3.0 IGO (CC BY 3.0 IGO) license, https:// creativecommons.org/licenses/by/3.0/igo/. Access to this work was provided by the University of Maryland, Baltimore County (UMBC) ScholarWorks@UMBC digital repository on the Maryland Shared Open Access (MD-SOAR) platform.

Please provide feedback

Please support the ScholarWorks@UMBC repository by emailing scholarworks-group@umbc.edu and telling us what having access to this work means to you and why it's important to you. Thank you. 


\title{
Women's Sexual Arousal: Effects of High Alcohol Dosages and Self-Control Instructions
}

\author{
William H. George, \\ University of Washington \\ Kelly Cue Davis, \\ University of Washington \\ Julia R. Heiman, \\ The Kinsey Institute \\ Jeanette Norris, \\ University of Washington \\ Susan A. Stoner, \\ Talaria, Inc \\ Rebecca L. Schacht, \\ University of Washington \\ Christian S. Hendershot, and \\ University of Toronto \\ Kelly F. Kajumulo \\ University of Washington
}

\section{Abstract}

The basic relationship between alcohol and women's sexual arousal - especially genital arousal received little research attention for nearly 30 years (e.g. Wilson \& Lawson, 1978) until very recently (e.g. George et al., 2009). To investigate hypotheses based on earlier findings and Alcohol Myopia Theory (AMT), two experiments evaluated the effects of high blood alcohol concentrations (BACs) and arousal instructional demands on indices of vaginal responding and self-reported sexual arousal. In Experiment 1, self-control instructions to maximize (versus suppress) arousal increased peak and average Vaginal Pulse Amplitude (VPA) change. Selfcontrol also interacted with a target BAC of .08\% (versus .00\%) to influence latency to peak arousal onset: Intoxicated women instructed to maximize showed a shorter latency to peak arousal than did intoxicated women instructed to suppress; however, sober women showed the same pattern. Also, in Experiment 1, the target BAC of .08\% had no effect on VPA or subjective arousal measures. In Experiment 2, a target BAC of .10\% (versus .00\%) attenuated peak change and average change in VPA, but this dosage had no effects on latency to peak achieved arousal, or on subjective arousal. Instructions to maximize arousal (versus no instruction) had no effect on any arousal measures. Overall, among young moderate drinking women, alcohol had attenuating

\section{(C) 2010 Elsevier Inc. All rights reserved.}

Correspondence regarding this article should be addressed to William H. George, Department of Psychology, Box 351525, University of Washington, Seattle, WA, 98195. Electronic mail may be sent to bgeorge@u.washington.edu.

Publisher's Disclaimer: This is a PDF file of an unedited manuscript that has been accepted for publication. As a service to our customers we are providing this early version of the manuscript. The manuscript will undergo copyediting, typesetting, and review of the resulting proof before it is published in its final citable form. Please note that during the production process errors may be discovered which could affect the content, and all legal disclaimers that apply to the journal pertain. 
effects but only at the higher dosage. Maximize versus suppress instructions about arousal had predicted effects on arousal and interactive effects on latency, but only at the lower dosage. The findings highlight the importance of dosage and contextual factors in alcohol's impact on the variability of women's sexual responding.

Research links alcohol to a variety of women's sexual outcomes (see reviews by Crowe \& George, 1989; George \& Stoner, 2000; Norris, 1994) including sexual functioning (e.g., Sobczak, 2009) and sexual risk taking (e.g., Norris, Masters et al., 2004). Women's sexual arousal during states of acute intoxication is relevant to and perhaps instrumental to understanding the link between alcohol and sexual outcomes, yet has received limited attention. A goal of this research was to extend the experimental data available for delineating alcohol's high dosage effects on women's sexual arousal: self-reported arousal and genital response. A second goal was to evaluate self-control conditions inspired by an Alcohol Myopia Theory (AMT) account of alcohol's attentional effects on women's selfreported sexual arousal.

\section{Alcohol and Self-Reported Sexual Arousal}

It has become well established through controlled experimentation that acute alcohol intoxication heightens women's self-reported sexual arousal. In two initial studies utilizing erotic films depicting explicit scenes of heterosexual intercourse, researchers found that increases in both blood alcohol concentration (BACs of .001, .025, .049, .079 $\mathrm{mg} \%$ ) (Wilson \& Lawson, 1976) and perceived intoxication (at a BAC of .04 $\mathrm{mg} \%$ ) (Wilson \& Lawson, 1978) were associated with increased self-reported sexual arousal. Malatesta et al. (1982) also reported that increasing dosage (particularly BACs of $.05 \mathrm{mg} \%, .075 \mathrm{mg} \%$ ) was associated with greater self-reports of arousal and orgasmic pleasure. Davis et al. (2006) found that alcohol (BAC $=.06 \mathrm{mg} \%$ ) increased women's self-reported arousal to an eroticized third-person vignette depicting a man making sexual advances on a woman forcefully. Also, this effect was moderated by expectancy such that alcohol-increased sexual arousal was more evident for women who highly endorse the expectancy that "alcohol enhances sex." Using a similar vignette, Norris, Davis et al. (2004) found no alcohol effect, but reported an interaction indicating increased arousal for women who received placebo drinks and who more highly endorsed the expectancy that "alcohol enhances sex." George et al. (2009; Study 3) found that alcohol (BACs $=.00 \mathrm{mg} \%, .06 \mathrm{mg} \%, .08 \mathrm{mg} \%, .10 \mathrm{mg} \%$ ) increased self-reported arousal to an eroticized second-person vignette of a consensual interaction in which the participant was instructed to project herself into the role of the woman in the vignette. Norris, Stoner, Hessler, Zawacki, Davis, et al. (2009) also found that alcohol (BACs $=.00 \mathrm{mg} \%, .04 \mathrm{mg} \%, .08 \mathrm{mg} \%$ ) increased arousal to an eroticized vignette of consensual sex. Examining effects in the context of sexual abuse history, Schacht et al. (2007) found that alcohol (BAC $=.08 \mathrm{mg} \%$ ) increased arousal to erotic films among women previously victimized by sexual abuse; but alcohol decreased arousal for non-victims. In a study of women who reported a history of either childhood sexual abuse, adult sexual assault, or no abuse, Schacht, George et al. (2010) found alcohol (BACs $=.00 \mathrm{mg} \%, .06 \mathrm{mg}$ $\%, .08 \mathrm{mg} \%, .10 \mathrm{mg} \%$ ) increased arousal to an eroticized vignette, regardless of abuse status. In contrast, Gilmore et al. (2010) found no effect for alcohol (BAC $=.10 \mathrm{mg} \%$ ) on women's self-reported arousal to the Schacht et al. (2007) film clips, regardless of abuse status.

In mixed gender experiments, women (and men) exhibited alcohol-induced increases in selfreported arousal. McCarty et al. (1982) found that participants reported greater arousal to erotic slides after they had received an alcoholic drink $(\mathrm{BAC}=.045 \mathrm{mg} \%)$ that they did not know contained alcohol rather than a known non-alcoholic beverage that they knew contained no alcohol. Abbey et al. (2006) found that participants reported greater arousal to 
a video depicting sexual advances, after they had received an alcoholic $(\mathrm{BAC}=.08 \mathrm{mg} \%$ ) rather than a known non-alcoholic beverage. Prause, Staley, and Finn (2011) found that alcohol (BACs $=.025$ and $.08 \mathrm{mg} \%$ ) increased self-reported sexual arousal across multiple exposures to both erotic and neutral films. George et al. (2009; Study 1), found that alcohol at a high dosage $(\mathrm{BAC}=.08 \mathrm{mg} \%)$ - but not at a low dosage $(\mathrm{BAC}=.04 \mathrm{mg} \%)-$ increased arousal to an eroticized vignette. In an experiment manipulating alcohol $(\mathrm{BAC}=.08 \mathrm{mg} \%)$ and BAC limb (BAC was either ascending or descending), Davis et al. (2009) found support for a model indicating direct effects for alcohol on perceived intoxication, which, in turn, led to greater arousal among participants exposed to an eroticized vignette. Furthermore, individuals with ascending BACs reported greater perceived intoxication, which led to greater perceived arousal, than individuals with descending BACs, confirming speculation that intoxication phase- as well as dosage- is an important parameter in understanding alcohol's effects on sexual arousal (George \& Stoner, 2000).

In sum, with little exception (Gilmore et al., 2010;Norris, Davis et al., 2004), alcohol increased women's self-reported sexual arousal under controlled conditions in 13 of 15 experiments. This alcohol enhancement effect also occurred when women were asked to appraise the sexual potential of a vignette (e.g. Norris, Stoner, Hessler, Zawacki, George et al., 2009). The effect has been observed with varying dosages, although generally it is more evident with higher dosages. It has also been observed with varying stimulus paradigms: Erotic slides, film depictions of sexual advances, film depictions of explicit nude intercourse, eroticized third-person vignettes of nonconsensual sex, and eroticized secondperson vignettes of consensual sex.

\section{Alcohol and Genital Responding}

Paradoxically, there is considerable evidence indicating that alcohol can have opposite - i.e. attenuating - effects on physiological indices of vaginal responding. This paradox is not surprising given the low agreement generally between women's self-report and genital measures across all sexual arousal studies (not just alcohol studies). In Chivers et al.'s (2010) meta-analysis of studies examining the correspondence between self-report versus genital arousal, self-report and genital measures correlated substantially lower for women ( $\mathrm{r}$ $=.26)$ than for men $(\mathrm{r}=.66)$. Therefore, although paradoxical, it is not contradictory empirically that alcohol can have opposite effects on women's self-reported versus genital arousal.

Eight studies have examined alcohol's effects on genital responding. Two initial studies (Wilson \& Lawson, 1976; 1978) evaluated the effects of acute intoxication on vaginal pulse amplitude (VPA). Wilson and Lawson (1976) found a negative linear relationship between dosage and VPA. Wilson and Lawson (1978) found that alcohol lowered vaginal pulse pressure. Malatesta et al. (1982) assessed vaginal blood volume, but did not report on it as an arousal indicator. Instead, they combined vaginal and behavioral data to indicate orgasm latency, revealing that increased dosage linearly increased latency, suggesting decreased genital arousal. In our own laboratory, findings have been mixed. George et al. (2009; Study 3 ) found that alcohol decreased VPA to an eroticized consensual second-person vignette. Gilmore et al. (2010) found that alcohol (BAC $=.10 \mathrm{mg} \%$ ) decreased VPA to erotic films. However, in studies distinguishing victimized and non-victimized women, alcohol had no effect on VPA to erotic films (Schacht et al., 2007; BAC $=.08 \mathrm{mg} \%$ ) or to a vignette (Schacht, George et al., 2010; BAC $=.10 \mathrm{mg} \%$ ). In the only mixed gender study reported, alcohol had no effect on VPA to erotic films (Prause et al., 2011). Thus, across eight studies, all but three (Prause et al., 2011; Schacht et al., 2007; Schacht, George et al., 2010) revealed that alcohol reduced genital arousal with the effect being more evident at higher dosages. 


\section{Alcohol Myopia Theory and Attentional Processes}

There is no unifying explanation of alcohol's enhancement effect on women's self-reported arousal and its attenuation effect on genital arousal. When the alcohol dosage is greater than .08 , it is generally understood that alcohol's physiological effects exert a greater influence than its expectancy (learned) effects. At these higher dosages where enhancement effects on self-reported arousal have been most observed and where alcohol's physiological effects are indisputably and determinatively at play, Alcohol Myopia Theory (AMT) offers the most applicable and compelling explanation. One of alcohol's most reliable physiological effects is that it impairs cognitive information processes, restricting attention allocation to a narrower range of behaviorally influential cues (Giancola, 2002). AMT builds on this well-established finding and asserts that intoxication narrows the drinker's attention to a restricted set of salient cues prompting behavioral outcomes. Generally, in appetitive situations such as sexual encounters, the impelling or "go" cues tend to be higher in salience than inhibiting or "stop" cues. As a result the drinker's responses are more determined by narrowed (myopic) attention to high salience impelling cues in the moment than by lower salience inhibiting cues. Thus, an intoxicated person compared to a sober person would likely attend most to the sexual stimuli and her own initial arousal changes and - as a consequence - would thereby experience and report more arousal. In accord with his line of reasoning, any manipulation fostering greater salience of or attention to erotic stimuli and/or the arousal response should consequently intensify the alcohol effect on self-reported arousal.

\section{Attentional Processes and Self-Control of Arousal}

Attentional processes also play a central role in our understanding of sexual arousal generally (Barlow, 1986). Furthermore, "the relevance of attentional processes to sexual arousal was perhaps first established by the early studies on voluntary control of sexual arousal" (italics added, de Jong, 2009, p. 239). In studies not involving alcohol, voluntary arousal control has been clearly demonstrated in women. Cerny (1978) and Hoon (1980) found that sexually functional women exhibited greater physiological vaginal responding and subjective arousal when instructed to increase rather than to decrease arousal. Likewise, Laan et al. (1993) found that sexually functional women exhibited greater VPA and subjective arousal when given a "performance demand" instruction to "try to become as aroused as possible ... and try to maintain it as long as you can" than when given no demand instruction. Beck and Baldwin (1994) instructed sexually functional women to "become as aroused as possible" and alternately to "suppress arousal." They found that $40 \%$ of women were able to control physiological arousal effectively and that these successful participants were able to identify more cognitive attentional strategies used to achieve arousal control than were unsuccessful participants. Similarly, Otto et al. (2009) reported that women instructed to maximize their arousal did so cognitively by attending to the erotic stimuli and their own arousal.

The centrality of attentional processes to understanding both sexual arousal generally and alcohol's enhancement effects on self-reported arousal - vis-a-vis AMT - indicates that experimental manipulations intended to heighten attention to erotic response are especially germane to elucidating alcohol's enhancement effect. Arousal self-control manipulations therefore constitute an important yet underutilized tool in evaluating alcohol-arousal hypotheses. Specifically, evidence that arousal self-control is mediated by attentional processes - as are AMT accounts of enhancement effects - suggests that alcohol effects should vary with arousal self-control conditions. 


\section{Present Study}

We evaluated four initial hypotheses about alcohol effects on self-reported and genital arousal. Based on previous findings, we hypothesized that women would report subjectively and exhibit physiologically greater arousal under self-control instructions to maximize arousal (Hypothesis 1) and would report greater arousal under alcohol versus no-alcohol conditions (Hypothesis 2). Third, consistent with AMT, we expected an alcohol by selfcontrol interaction on self-reported arousal (Hypothesis 3). Under maximize instructions, we expected women to attend more to the erotic stimuli and their own arousal. The myopia effect of alcohol was expected to enhance this attentional focus such that women in the maximize-alcohol condition would report greater arousal than maximize-sober counterparts. Finally, conversely and yet congruent with past findings indicating alcohol's opposite effects on self-reported versus genital arousal, we hypothesized that alcohol would decrease VPA (Hypothesis 4).

\section{Study 1 \\ Method}

Participants-Women $(n=78)$ were recruited through flyers, newspaper advertisements, and letters, which stated that the study involved "social drinking and decision-making." Potential participants (Ps) called the laboratory and were told that procedures included the use of sexually explicit films and physiological measures of sexual arousal, and were screened to determine eligibility. Inclusion criteria were that the woman had to be (a) between the ages of 21 and 35; (b) interested in dating opposite-sex partners; (c) not currently in a committed dating relationship; and (d) a moderate drinker. These criteria ensured that all participants would find the experimental story relevant to their current dating status and lifestyle. Exclusion criteria were current problem drinking or a history of problem drinking1 and/or currently taking medications or having a health condition contraindicated with alcohol consumption. Ps received $\$ 15$ per hour for study participation.

Mean age was 25.3 years $(S D=3.5)$. Ps were predominantly European-American $(80.5 \%)$; $3.0 \%$ were Asian, $6.2 \%$ were African-American, $4.6 \%$ were Latino, $3.1 \%$ were Native American, and $2.9 \%$ were multi-racial or other. Over one-half (72.1\%) were employed with $76.6 \%$ reporting an annual income of less than $\$ 31,000 /$ year. Ps' self-reported mean drinking level was $9.16(\mathrm{SD}=6.82)$ drinks per week. Fewer than one-half $(34.3 \%)$ were college students.

\section{Materials and measures}

Stimulus films-Ps viewed a sexually-neutral 2.5-minute long documentary film about birds, followed by a six-minute erotic film containing two segments, each depicting a different couple. Pilot testing established that these erotic segments induced equivalent selfreported increases in sexual arousal (George, 2004). Therefore, the segments were balanced in content and were equivalent in stimulus intensity. Both segments, which were from commercially available films, explicitly depicted consensual sexual activities (kissing, oral sex, and vaginal intercourse) between an adult man and an adult woman.

Physiological sexual arousal-Sexual arousal was measured using vaginal photoplethysmography (BioPac Systems, Inc., Santa Barbara, CA; model MP 150) and a

\footnotetext{
${ }^{1}$ Potential participants were excluded if they reported having been (a) told by a professional that they had problem with alcohol, (b) ever seriously concerned about their own drinking, or (c) treated or advised to seek treatment for drinking. They were also excluded if they had ever experienced any of the following after drinking alcohol: (a) fainting or seizure, (b) highly unusual flushing of the skin, or (c) severe or unusual psychological reaction. Finally, those who reported a positive family history for alcoholism were also excluded.
} 
tampon-sized Plexiglas vaginal probe (Behavioral Technology, Inc., Salt Lake City, UT). The probe, which contains an infrared light source and a photo cell, was inserted into the vagina. The depth and orientation of the probe was standardized between women by the placement of a plexiglass plate attached to the photoplethysmograph cable. The plexiglass plate was situated approximately $2.5 \mathrm{~cm}$ from the end of the probe and the probe was oriented to illuminate the anterior vaginal wall. The probed and plate were sanitized following each use with a glutaraldehyde solution (Cidex OPA, Advanced Sterilization Products, Irvine, CA). Changes in the amount of light backscattered from the vaginal wall as it becomes engorged with blood was measured and subsequently transformed into digital data for analysis. Vaginal pulse amplitude (VPA) was continuously sampled at a rate of 62.5 samples per second, and data were recorded using Acqknowledge software, version 3.7.2. Waveform data were visually inspected by an experienced investigator and movement artifacts were removed. Acqknowledge software was used to reduce the data to 25 samples per second, measure peak-to-trough differences for each pulse, and compute mean peak-totrough values for each 30-second interval. Remaining movement artifacts, defined as a $100 \%$ increase or decrease in VPA relative to the adjacent 30 -second intervals, were smoothed by averaging the values of the adjacent intervals (Schacht et al., 2007). The specific derived measures used here were self-reported ratings of sexual arousal, peak change in VPA, average change in VPA, and latency to peak VPA change.

Self-reported ratings of sexual arousal-Ps rated their level of sexual arousal after the neutral film and after the erotic film son a four-item seven-point Likert scale ( $1=$ no sexual arousal at all; $7=$ extremely sexually aroused). Items were adapted from previous research and included: (1) Overall, how much sexual arousal did you feel during the film clips? (Heiman, 1977); (2) To what extent did you feel sensation in your genitals during the film clips? (Heiman \& Rowland, 1983; Reynolds et al., 1988); (3) How much sexual warmth (in your genitals, breasts, and body) did you feel during the film clips? (Meston et al., 1998); and (4) To what extent did you feel sexually absorbed in the sensory components of the film clips? These four items were combined to form a sexual arousal scale with good inter-item reliability (Study 1 sample $M=4.61, S D=1.43$; Study 2 sample $M=4.87, S D=1.49 ; \alpha=$. 94).

Balanced Inventory of Desired Responding (BIDR)-This 40-item measure was used to assess social desirability. Items are based on a 7-point Likert scale, and include: I sometimes tell lies if I have to, I never read sexy books or magazines, and I sometimes drive faster than the speed limit. This measure has acceptable reliability $(\alpha=.83$; Paulhus, 1991; Study 1 sample $M=163.25, S D=31.21 ; \alpha=.80 ;$ Study 2 sample $M=160.67, S D=31.64 ; \alpha$ $=.80[$ this measure is calculated as a sum]).

Manipulation checks-To determine whether Ps had been aware of their assigned sexual arousal instructional set, manipulation checks were administered following the sexual arousal assessment. Ps were asked which instruction they had been given.

\section{Procedure}

Pre-experimental instructions-During the screening phone call, Ps were instructed to bring photo identification, not to drive to the laboratory, not to eat or consume caloric drinks for three hours before their appointments, and not to drink alcohol or use recreational or over-the-counter drugs for 24 hours before their appointments.

Initial procedures_-Each participant was conducted through the study procedures by a female experimenter. Upon arrival, each participant was escorted to a private room where the experimenter administered a blood alcohol level (BAL) test with a breathalyzer (Alco- 
Sensor IV, Intoximeters Inc., St. Louis, MO) to verify a zero reading. Ps with a positive reading, as well as Ps who reported that they were currently menstruating, were rescheduled and dismissed. After confirming that the participant had complied with all pre-experimental instructions, the experimenter obtained informed consent. Before alcohol administration, all Ps were given a pregnancy test (OsomhCG-Urine Test, Genzyme General Diagnostics, San Diego, CA) to ascertain that they were not pregnant. Afterwards, the $\mathrm{P}$ was left alone to complete background questionnaires.

Alcohol procedures and administration-Each $\mathrm{P}$ was randomly assigned to an alcohol condition and was weighed to determined the amount of 100-proof vodka needed to achieve a BAL of $.08 \%$ (dosage $=.82 \mathrm{ml}$ alcohol $/ \mathrm{kg}$ body weight). Drinks consisted of one part vodka to four parts fruit juice. Ps' BALs were tested every three minutes until they reached criterion (BAL $\geq .045 \%$ ), at which point they began the sexual arousal assessment. Ps' mean pre-assessment BAL was $.060(S D=.001)$. Ps took on average 17.00 minutes ( $S D$ $=9.00$ ) post-drinking to reach the target BAL. Ps in the no-alcohol condition drank a known-non-alcoholic volume of juice equivalent to what they would have received in the alcohol condition. A yoked control design was used to reduce error variance in intoxication levels by controlling for differences in the time it took individual alcohol Ps to reach the criterion BAL (Schacht, Stoner et al., 2010; Giancola \& Zeichner, 1997). Control Ps were yoked to an alcohol $\mathrm{P}$ who had already participated, and were breathalyzed and began the dependent measures at the same time intervals as their alcohol yoke.

Sexual arousal instructional set-Ps were randomly assigned to one of two instructional set conditions to either maximize or suppress their sexual arousal. Audio taped instructions were presented via headphones; therefore experimenters were blind to Ps' arousal instruction condition. After the neutral film and before the erotic film, Ps received audio instructions over headphones informing them of their arousal instruction and directing them to open an envelope containing a card reiterating this instruction. In the maximize condition, the instructions were to "try as much as possible to relax and maximize your arousal during the remainder of the experiment. We would like you to try and become as aroused as possible." In the suppress condition, the instructions were to "try as much as possible to suppress your sexual arousal during the remainder of the experiment. In other words, please keep from becoming sexually aroused." Women in both conditions reported using cognitive strategies to achieve success in regulating their arousal (Otto et al., 2009).

Sexual arousal assessment-Before providing beverages, the experimenter instructed the $\mathrm{P}$ on the proper placement of the plethysmograph. After Ps consumed their beverage and reached criterion BAL, the experimenter $(\mathrm{E})$ restated instructions by intercom on placement of the vaginal probe, and $\mathrm{P}$ inserted the probe. E monitored the physiological signal via computer in a separate room, and, if needed, instructed $\mathrm{P}$ by intercom to readjust the probe. After obtaining a 1-minute baseline reading, the participant was shown the neutral film clip. Then E returned to the experimental room to obtain a final pre-arousal BAL assessment using a hand-held breathalyzer (Alco-Sensor IV, Intoximeters Inc., St. Louis, MO). After E left the room, the participant was shown the erotic film, rated their self-report level of sexual arousal immediately after film viewing, and was subsequently instructed by intercom to remove the probe and to get dressed. E then returned to and administered a postexperimental BAL measurement.

Detoxification and debriefing-Control Ps were debriefed, paid, and released upon completion of the experiment. Alcohol Ps were escorted to another room, where they remained until their BAL dropped below .03, at which point they were debriefed, paid, and 
released. During debriefing, all Ps were given the opportunity to ask questions and to discuss any discomfort they might have experienced during the protocols.

\section{Study 1 Results}

Data analytic strategy-Analysis of variance (ANOVA) tests examined the effects of alcohol dose (control vs. alcohol) and arousal instructional set condition (suppress vs. maximize) on the dependent variables. To assess the vaginal photoplethysmography results, three variables were examined. The first variable, peak VPA difference score, was computed by subtracting Ps' lowest 30 seconds of VPA during the neutral stimulus from their highest 30 seconds of VPA during the erotic stimulus. The second variable, mean VPA difference score, was computed by subtracting Ps' mean VPA during the neutral stimulus from their mean VPA during the erotic stimulus. The third variable, latency to peak arousal, was computed by determining the number of seconds from the beginning of the erotic stimulus until the peak level of arousal during the erotic stimuli with the peak VPA entered as a covariate in order to control for differences in achieved peak arousal levels. Self-reported sexual arousal during the erotic stimuli was examined with social desirability score entered as a covariate. Identical analytic strategies were used in Studies 1 and 2. Data from 10 Ps in Study 1 and 10 Ps in Study 2 were incomplete due to data collection problems (including 2 for incomplete data [both in Study 1], 12 for movement artifacts rendering their VPA data un interpretable [5 in Study 1 and 7 in Study 2], and 6 for equipment failure [3 in Study 1 and 3 in Study 2]). These 20 Ps were not included in data analyses, resulting in a final sample size of 68 for Study 1 and 64 for Study 2.

Manipulation checks-Three Ps eliminated from the Study 1 sample for the aforementioned reasons also failed the manipulation checks concerning instructional set.

Self-reported sexual arousal-Consistent with Hypothesis 1, there was a main effect for arousal self-control condition, $F(1,67)=4.77, p<.05$. Ps instructed to maximize arousal reported greater arousal than did Ps instructed to suppress arousal. Contrary to Hypotheses 2 and 3, there were no significant alcohol main effects or interactions on self-reported sexual arousal. Social desirability score was not a significant covariate.

Genital arousal: Peak VPA and average VPA difference scores-Consistent with Hypothesis 1, analyses revealed a main effect for arousal self-control condition on peak VPA difference score, $F(1,68)=10.98, p<.01$, and average VPA difference score, $F(1,68)$ $=8.00, p<.01$. Ps instructed to maximize arousal achieved a greater peak VPA change and average VPA change from baseline than did Ps instructed to suppress arousal. Means are presented in Table 1. Contrary to our second and third hypotheses, there were no alcohol main effects or interactions on peak or average VPA scores. Contrary to our fourth hypothesis, alcohol did not decrease VPA.

Latency to peak arousal-As illustrated in Figure 1, the two-way interaction of alcohol and self-control conditions was significant, $F(1,68)=4.70, p<.05$. Post hoc analyses indicated that, intoxicated Ps instructed to maximize achieved peak VPA from baseline significantly faster than did intoxicated Ps instructed to suppress; sober Ps did not show this differential pattern. The covariate of VPA was not significant. There were no other significant effects.

\section{Discussion}

Study 1 findings provided mixed support for our four initial hypotheses. With regard to selfreported arousal, the hypothesized self-control effect was obtained (Hypothesis 1). However, while the hypothesized alcohol main (Hypothesis 2) and interaction effects (Hypothesis 3) 
were not evident on self-reported arousal, the predicted interaction was evident on one of three genital measures. Also, surprisingly, the hypothesized alcohol attenuation effect on genital arousal was not evident (Hypothesis 4).

Consistent with Hypothesis 1, the arousal instructions had a significant effect on physiological and self-reported arousal. Women in the maximize condition showed greater self-reported arousal and exhibited greater peak and mean VPA increases than did women in the suppress condition. These main effects are consistent with previous work showing that, despite initial speculations of limited conscious control over genital responding (e.g. Hatch, 1981), women can exert control over sexual arousal (de Jong, 2009). As demonstrated earlier (Beck \& Baldwin, 1994), this self-control is achieved via cognitive strategies. Women in the maximize condition were more focused on their own arousal and on the erotic aspects of the stimuli than were women in the suppress condition who were more focused on non-erotic aspects of the stimuli and lab surroundings (Otto et al., 2009). Nevertheless, a shortcoming in interpreting this effect clearly is the absence of a neutral point. Therefore, it was not possible to isolate whether maximize instructions increased arousal or suppress instructions decreased arousal or both. An aim of Study 2 was to compare the maximize condition to a neutral condition to clarify the increase in arousal observed in this study.

The absence of the hypothesized alcohol-induced increase in self-reported arousal and decrease in genital arousal were surprising. These effects have been robust in previous selfreport (13 of the 15 studies) and genital (5 of the 8 studies) findings. The current dosage was within the range of dosages in which these effects have been observed previously. These discrepancies could potentially be due to unspecified methodological differences unique to this study. Nevertheless, it is important to pursue whether the effects emerge in the current paradigm with higher dosage; this was another aim of Study 2.

In the absence of an alcohol interaction with instruction, there was no support provided for the AMT hypothesis on self-reported arousal. However, considering the lack of an alcohol effect on self-reported arousal and that AMT processes tend to manifest as dosage increases, probing the AMT hypothesis at a higher dosage was warranted.

Alcohol, although there were no main effects, did have an interactive effect on one of the three genital measures: latency to peak VPA. Intoxicated women reached peak arousal faster in the maximize condition than in the suppress condition, whereas sober women did not show this differential pattern. This pattern - while not predicted for VPA measures because of the robust alcohol suppressant effect observed previously on genital measures - is, in fact, consistent with the AMT hypothesis. That intoxicated-maximize women reached peak VPA sooner than intoxicated-suppress women is consistent with experiencing myopic attentional focus on arousal. This is compared to sober women presumably not experiencing alcoholinduced myopia and who accordingly exhibited equivalent latencies. It will be important to examine whether this AMT pattern on VPA latency - although modest in effect size - recurs at a higher dosage.

\section{Study 2}

The aims of Study 2 were to clarify the self-control effects by evaluating whether the maximize condition would generate greater arousal than a neutral condition (Hypothesis 5) and to evaluate whether the alcohol effects predicted in Hypotheses 2, 3, and 4 emerge with increased dosage. Ethical and IRB constraints prevented examining a BAL higher than . $10 \%$. 
Study 2 was identical to Study 1 with the exception of the following.

Participants-Seventy-four women were recruited. Ps' mean age was 25.2 years $(S D=$ 4.2). Ps were predominantly European-American (79.7\%); $7.8 \%$ were Asian, $3.1 \%$ were African-American, $3.2 \%$ were Hispanic, and $6.2 \%$ were multi-racial or other.

Approximately two-thirds (68.3\%) were employed, with 56.3\% reporting an annual income of less than $\$ 31,000 /$ year. Ps' self-reported mean drinking level was $10.8(\mathrm{SD}=7.5)$ drinks per week on average. Less than one-half $(46.0 \%)$ were college students.

Sexual arousal instructional set-Ps were randomly assigned to a maximize-arousal instructional condition or a no-instruction control condition. In the control condition, the instructions were told "remember: try to stay as still as possible."

Alcohol-Each P was weighed to determine the amount of 190-proof grain alcohol needed to achieve a BAL of $.10 \%$ (dosage $=1.25 \mathrm{ml}$ alcohol $/ \mathrm{kg}$ body weight). Drinks consisted of one part alcohol to six parts fruit juice. In order to ensure that the assessment took place on the ascending limb of alcohol intoxication, Ps' BALs were tested every three minutes until they reached criterion (BAL $\geq .06 \%$ ), at which point they began the sexual arousal assessment. Ps took on average 26 minutes $(S D=10)$ post-drinking to reach the target BAL. Mean pre-arousal assessment BAL was .083\% $(S D=.011)$. Ps in the no-alcohol condition drank a known-non-alcoholic volume of juice equivalent to what they would have received in the alcohol condition.

\section{Study 2 Results}

Manipulation check-One P eliminated from the Study 2 sample for equipment failure also failed the manipulation check concerning instructional set.

Self-reported sexual arousal—Contrary to our hypotheses, there were no main effects or interactions. Social desirability score was not a significant covariate.

Genital arousal: Peak VPA and average VPA difference scores-Contrary to Hypothesis 1, there was no effect for self-control conditions on VPA. Consistent with Hypothesis 4 , there were alcohol effects on peak, $F(1,64)=6.29, p<.05$, and average VPA difference scores, $F(1,64)=4.97, p<.05$. Intoxicated Ps experienced significantly smaller peak and average VPA difference scores than did sober Ps. See Table 2 for means, standard deviations, and effect sizes. There were no other significant main effects or interactions.

Latency to peak arousal-For latency to peak arousal, there were no significant main effects or interactions. The covariate of peak genital response was not significant.

\section{Discussion}

Study 2 findings provided little support for our hypotheses. With regard to self-reported arousal, the hypothesized self-control effect that occurred when the maximize condition was compared to a suppress condition did not reoccur when it was compared to a neutral condition (Hypothesis 1b). Again, as in Study 1, there were no alcohol main (Hypothesis 2) or interaction (Hypothesis 3) effects on self-reported arousal. Consistent with Hypothesis 4, increasing the alcohol dosage to .10 did result in the alcohol-induced attenuation effect on genital arousal.

Contrary to Hypothesis 5, the self-control conditions had no effect on vaginal arousal or self-reported arousal. This is inconsistent with Study 1 findings and previous work. 
However, each earlier study compared self-control conditions of greater contrast. Laan et al. (1993) used perhaps a more potent increase-arousal instruction compared to this study's by providing a "performance demand" instruction to "try to become as aroused as possible ... and try to maintain it as long as you can" which was contrasted with a no demand instruction. Three studies contrasted increase-arousal instructions with instructions either to decrease (Cerny, 1978; Hoon, 1980) or suppress (Beck \& Baldwin, 1994) arousal. In the present study, the neutral and maximize conditions may not have represented sufficient contrast. The Study 1 maximize and suppress conditions likely yielded increases and decreases respectively in arousal relative to an implied neutral point. However, when an actual neutral point was operationalized in Study 2, any arousal increase fostered by the maximize condition was insufficient to attain significance relative to the neutral condition. In addition, the possibility that the alcohol dosage was so high that it overrode any effects for self-control condition cannot be ruled out.

Again, as in Study 1, alcohol did not enhance self-reported sexual arousal (Hypothesis 2). The failure to detect an alcohol enhancement effect on self-reported arousal is not consistent with previous work. The reason for this discrepancy is unclear. However, it should be noted that this is the only evaluation of the enhancement effect in which participants were given explicit instructions to control their arousal response. Conceivably, the effort to control their arousal overrode or some how disrupted the usual alcohol enhancement effect observed in 13 of 15 previous studies. Accordingly, in the absence of an enhancement effect to explain, no support was garnered for the AMT hypothesis on self-report arousal (Hypothesis 3) at either dosage level. Interestingly, the only indication of the support for the AMT was on the Study 1 latency measure, which can be interpreted as a more implicit indicator of arousal than the magnitude indicators (for which participants were given explicit control instructions). Thus, in contrast to arousal magnitude indicators where there was an explicit instruction to exert conscious effort at managing their response, the hypothesized AMT effect was more discernible on an implicit indicator. This speculation warrants further research.

As hypothesized (Hypothesis 4), alcohol attenuated women's genital arousal magnitude. Thus, while alcohol had no impact on genital arousal latency - as it did interactively in Study 1, alcohol had a clear attenuation effect on magnitude. Compared with sober controls, women dosed to a target BAL of .10\% exhibited less peak and average VPA change from baseline. These findings are consistent with most previous studies. Altogether, now six of 10 studies conducted to date have shown that acute alcohol intoxication attenuates women's genital response. Among the four experiments reporting null findings, three utilized dosages at .08 or below (current paper - Study 1; Prause et al., 2011; Schacht et al., 2007) In the one study in which attenuation did not occur at the .10\% dosage (Schacht, George et al., 2010), women were responding to a vignette rather than a film, suggesting that the effect may be more reliably discernible with visual stimuli.

\section{General Discussion}

The present findings established that women can exert self-control over the magnitude of their sexual arousal response - both self-report and genital indices. Based on using betweensubject designs and the absence of a maximize effect relative to a neutral point, this selfcontrol effect seemed characterized by divergent alcohol-induced increases and decreases in the contrasting maximize versus suppress conditions. As in previous demonstrations of women's arousal self-control (e.g. Beck \& Baldwin, 1994), this control was accomplished via utilization of cognitive strategies (Otto et al., 2009) involving increased focus on arousing versus non-arousing aspects of the situation. It is striking that this arousal control was evident regardless of moderate (.08\%) intoxication. To our knowledge, this is the first 
demonstration of women's arousal control while under acute alcohol intoxication. However, while women at the lower dosage were able to successfully control their arousal levels as effectively as sober women, women at the higher dosage could not do so. The fact that women can effectively control their arousal level combined with the fact that subjective arousal mediates alcohol's effects on sexual risk-taking (George et al., 2009) could suggest important prevention implications. For instance, prevention intervention content aimed at teaching women to reduce arousal in risky sexual situations - even those involving low to moderate intoxication - could potentially prove a valuable strategy for mitigating sexual risk taking.

The present findings replicated and also clearly delimited the alcohol attenuation effect on vaginal arousal. Identical studies with men also showed that this (.10\%) dosage had an attenuation effect on peak change in penile tumescence (George et al., 2006). Thus, it seems clear that a decidedly high alcohol dosage (.10\%) attenuates heterosexual women's and men's genital responding to erotic conditions characterized by film depictions of explicit heterosexual intercourse. As Crowe and George noted (1989), this attenuation effect is independent of alcohol's enhancement (or null) effects on self-reported arousal (there does not appear to be any robust evidence in the literature that alcohol decreases self-reported arousal). Mechanisms underlying the attenuation effect on genital arousal are not well understood (Frohmader et al., 2010). After failing to detect attenuation in sleeping men and canines at dosages higher than (.10\%), Morlet et al. (1990) suggested that attenuation observed elsewhere in wakeful men is more likely attributable to psychological mechanisms necessitating awareness rather than physiological mechanisms. While research with women has suggested that hormonal responding may constitute important mechanisms in attenuation (Ericksson et al., 1994; Pfaus et al., 2010), this has not been shown directly and psychological mechanisms have not been ruled out.

Another important note regarding alcohol-induced attenuation of genital responding in women concerns the overall low correspondence between genital and self-reported arousal. The attenuation finding on VPA we observed in Study 2 was concurrent with a null finding on self-reported arousal. This apparent disconnect between these response domains is consistent with previous findings (e.g. George et al., 2009). These patterns are in keeping with the low correspondence between physiological and subjective arousal measures for women (Chivers et al., 2010).

\section{Strengths and Limitations}

A particular strength of this study is that alcohol factors, including dosage, limb, and absorption times were tightly controlled through rigorous procedures involving consistent BAL monitoring, specific BAL criterion starting points, and yoked controls. Another strength was present in our sampling of both students and non students, bolstering the generalizability of our findings. Limitations include the lack of an alcohol expectancy (placebo) condition, the moderate level drinking status and young age of the sample, the prospect of volunteer bias, power considerations, and constraints on VPA interpretation. First, previous work has shown that expectancy set - the mere belief that one has consumed an alcoholic drink - has little effect onwomen's self-reported and physiological arousal. Because of the difficulty of convincing placebo participants that they have received a high dose of alcohol, and because our specific interest was in high dosage effects, we did not evaluate alcohol expectancy set or individual differences in a priori alcohol expectancies (beliefs individuals hold about the effects of alcohol). At high dosages, physiological effects were expected to override any expectancy effects. Given the high BAC levels and the direction of the effects, it is unlikely that expectancies accounted for our findings. Second, sample characteristics, such as exclusion of heavy problem drinkers, limit the generalizability of our findings. Third, volunteers for sexual psychophysiological studies 
tend to have more sexual experience and liberal sexual attitudes than non-volunteers (Strassberg \& Lowe, 1995). This tendency constrains generalizability; therefore our findings should be interpreted accordingly. Fourth, while these sample sizes (study $1 n=68$; study 2 $n=64$ ) are among the largest reported for experiments evaluating the effects of acute alcohol consumption on women's psychophysiological genital responding (the largest to date appears to be Gilmore et al., 2010, $n=130$ ), we cannot rule out the possibility that our sample and design were underpowered for detecting small effect sizes. Therefore, future studies with larger samples are warranted for evaluating the possibility that alcohol's effects on women's sexual responding may be moderated by additional factors yielding interactions characterized by small effect sizes. Finally, while VPA has been shown to be a valid and reliable measure of sexual arousal, it suffers from being a relative measure without an absolute baseline (e.g. Heiman \& Maravilla, 2007). Between-subjects comparisons are less reliable than within-subjects comparisons and thus require computation of a within-subjects difference score. However, the meaning of VPA difference scores - in terms of the physiological processes represented - is not fully understood and warrants caution in interpretation of VPA findings, particularly in light of evidence of systematic typological differences in VPA responding (Levin \& Wylie, 2008).

\section{Conclusions}

High dosage $(.10 \mathrm{mg} \%)$ alcohol attenuated genital arousal in a sample of relatively young moderate drinking women. Our lower dosage $(.08 \mathrm{mg} \%)$, which is widely recognized as substantially intoxicating in that it is the legal criterion for drunk driving and it is widely associated with generalized impairment (e.g. Fell \& Voas, 2006), had no effect. Thus, looking across these and other studies it appears reasonable to conclude that until the BAC exceeds $.08 \mathrm{mg} \%$, women are likely to exhibit an arousal response pattern characterized by enhanced self-reported sexual arousal, no alcohol-induced diminution in genital arousal, and an effective capability to control their arousal response. This profile - combined with (1) the understanding that alcohol expectancies can moderate women's sexual responding (e.g. Norris, Masters et al., 2004), (2) the AMT-theorized role of intoxication for fostering selective attention to sexually impelling situational cues, and (3) evidence that the mere presence of alcohol cues can heighten sexual inferences and appraisals (e.g. George \& Stoner, 2000) - suggests that women's post-drinking sexual responses are subject to considerable psychological variability and are not dictated by physiological imperatives or limits. However, this variability apparently decreases at higher dosages where it appears that intoxication effects may become less conducive to positive sexual responding and experience. Potentially, physiology related to high alcohol levels may overwhelm the sexuality-related response systems. Given heightened attention to alcohol effects on women's sexual activities (e.g., Norris, Davis et al., 2004), such as HIV related sexual risk taking, more research into alcohol effects on these fundamental sexual responses is warranted. Diminished vaginal responding at the high dosage does not appear to curtail sexual risk taking (George et al., 2009), suggesting that many unanswered questions remain. In particular, research examining hormone responses as potential mediating mechanisms could prove valuable in further elucidating these relationships.

\section{Acknowledgments}

Appreciation is expressed to Dr. Erick Janssen for consultation in stimulus selection to Dr. Tina Zawacki, Reasons Project staff, and numerous undergraduate assistants for their aid in data collection. Conduct of this research was supported in part by a grant from the National Institute on Alcohol Abuse and Alcoholism (AA13565) to William H. George. 


\section{References}

Abbey, A.; Saenz, C.; Buck, PO.; Parkhill, MR.; Hayman, LW. The effects of acute alcohol consumption, cognitive reserve, partner risk, and gender on sexual decision making; Journal of Studies on Alcohol. 2006. p. 113-121.http://www.jsad.com/

Barlow DH. Causes of sexual dysfunction: The role of anxiety and cognitive interference. Journal of Consulting \& Clinical Psychology. 1986; 54:140-148. [PubMed: 3700800]

Beck JG, Baldwin LE. Instructional control of female sexual responding. Archives of Sexual Behavior. 1994; 23:665-684. [PubMed: 7872861]

Cerny JA. Biofeedback and the voluntary control of sexual arousal in women. Behavior Therapy. 1978; 9:847-855.

Chivers ML, Seto MC, Lalumiere ML, Laan E, Grimbos T. Agreement of self-reported and genital measures of sexual arousal in men and women: A meta-analysis. Archives of Sexual Behavior. 2010; 39:5-56. [PubMed: 20049519]

Crowe LC, George WH. Alcohol and human sexuality: Review and integration. Psychological Bulletin. 1989; 105:374-386. [PubMed: 2660179]

de Jong DC. The role of attention in sexualarousal: Implications for treatment of sexual dysfunction. Journal of Sex Research. 2009; 46:237-248. [PubMed: 19308846]

Davis KC, Norris J, George WH, Martell J, Heiman JR. Men's likelihood of sexual aggression: The influence of alcohol, sexual arousal, and violent pornography. Aggressive Behavior. 2006; 32:581589.

Davis, KC.; George, WH.; Norris, J.; Schacht, RL.; Stoner, SA.; Hendershot, CS.; Kajumulo, KF. Effects of alcohol and blood alcohol concentration limb on sexual risk-taking intentions; Journal of Studies on Alcohol and Drugs. 2009. p. 499-507.http://www.jsad.com/

Ericksson CJ, Fukunaga T, Lindman R. Sex hormone response to alcohol. Nature. 1994; 369:711. [PubMed: 8008063]

Fell JC, Voas RB. The effectiveness of reducing illegal blood alcohol concentration (BAC) limits for driving: Evidence for lowering the limit to .05 BAC. Journal of Safety Research. 2006; 37:233243. [PubMed: 16824545]

Frohmader KS, Pitchers KK, Balfour ME, Coolen LM. Mixing pleasures: Review of the effects of drugs on sex behavior in humans and animal models. Hormones and Behavior. 2010; 58:149-162. [PubMed: 20004662]

George WH. Gender differences in sexual arousal to erotic films: The mediating role of sexual sensation seeking. Sexuality and Disability. 2004; 22:80-81. [Abstract 311].

George WH, Davis KC, Norris J, Heiman JR, Schacht RL, Stoner SA, Kajumulo KF. Alcohol and erectile response: The effects of high dosage in the context of demands to maximize sexual arousal. Experimental and Clinical Psychopharmacology. 2006; 14:461-470. [PubMed: 17115874]

George WH, Davis KC, Norris J, Heiman JR, Stoner SA, Schacht RL, Hendershot CS, Kajumulo KF. Indirect effects of acute alcohol intoxication on sexual risk-taking: The roles of subjective and physiological sexual arousal. Archives of Sexual Behavior. 2009; 38:498-513. [PubMed: 18431618]

George WH, Stoner SA. Understanding acute alcohol effects on sexual behavior. Annual Review of Sex Research. 2000; 11:92-124.

Giancola PR. Irritability, acute alcohol consumption, and aggressive behavior in men and women. Drug and Alcohol Dependence. 2002; 68:263-274. [PubMed: 12393221]

Giancola PR, Zeichner A. The biphasic effects of alcohol on human physical aggression. Journal of Abnormal Psychology. 1997; 106:598-607. [PubMed: 9358690]

Gilmore AK, Schacht RL, George WH, Otto JM, Davis KC, Heiman JR, Norris J, Kajumulo KF. Assessing women's sexual arousal in the context of sexual assault history and acute alcohol intoxication. Journal of Sexual Medicine. 2010; 7:2112-2119. [PubMed: 20367775]

Hatch JP. Voluntary control of sexual responding in men and women: Implications for the etiology and treatment of sexual dysfunctions. Biofeedback \& Self Regulation. 1981; 6:191-205. [PubMed: 7260188] 
Heiman JR. A psychophysiological exploration of sexual arousal patterns in females and males. Psychophysiology. 1977; 14:266-274. [PubMed: 854556]

Heiman, JR.; Maravilla, KR. Female sexual arousal response using serial magnetic resonance imaging with initial comparisons to vaginal photoplethysmography: Overview and evaluation. In: Janssen, E., editor. The psychophysiology of sex. Bloomington, IN: Indiana University Press; 2007. p. 103-128.

Heiman JR, Rowland DL. Affective and physiological sexual response patterns: The effects of instructions on sexually functional and dysfunctional men. Journal of Psychosomatic Research. 1983; 27:105-116. [PubMed: 6683315]

Hoon EF. Biofeedback-assisted sexual arousal in females: A comparison of visual and auditory modalities. Biofeedback and Self-Regulation. 1980; 5:175-191. [PubMed: 6156712]

Laan E, Everaerd W, Van Aanhold MT, Rebel M. Performance demand and sexual arousal in women. Behaviour Research and Therapy. 1993; 31:25-35. [PubMed: 8417725]

Levin RJ, Wylie K. Vaginal vasomotion-its appearance, measurement, and usefulness in assessing the mechanisms of vasodilatation. Journal of Sexual Medicine. 2008; 5:377-386. [PubMed: 18042218]

Malatesta VJ, Pollack RH, Crotty TD, Peacock LJ. Acute alcohol-intoxication and female orgasmic response. Journal of Sex Research. 1982; 18:1-17.

Meston CM, Heiman JR, Trapnell PD, Paulhus DL. Socially desirable responding and sexuality selfreports. Journal of Sex Research. 1998; 35:148-157.

McCarty D, Diamond W, Kaye M. Alcohol, sexual arousal, and the transfer of excitation. Journal of Personality and Social Psychology. 1982; 42:977-988. [PubMed: 7108741]

Morlet A, Watters GR, Dunn J, Keogh EJ, Creed KE, Tulloch AGS, Lord DJ, Earle CM. Effects of acute alcohol on penile tumescence in normal young men and dogs. Urology. 1990; 35:399-404. [PubMed: 2336768]

Norris, J. Alcohol and female sexuality: A look at expectancies and risks; Alcohol Health \& Research World. 1994. p. 197-201.Retrieved from http://pubs.niaaa.nih.gov/publications/toc18-3.htm

Norris J, Davis KC, George WH, Martell J, Heiman JR. Victim's response and alcohol-related factors as determinants of women's responses to violent pornography. Psychology of Women Quarterly. 2004; 28:59-69.

Norris J, Masters NT, Zawacki T. Cognitive mediation of women's sexual decision making: the influence of alcohol, contextual factors, and background variables. Annual Review of Sex Research. 2004; 15:258-296.

Norris J, Stoner SA, Hessler DA, Zawacki T, Davis KC, George WH, Morrison DM, Parkhill MR, Abdallah DA. Influences of sexual sensation seeking, alcohol consumption, and sexual arousal on women's behavioral intentions related to having unprotected sex. Psychology of Addictive Behaviors. 2009; 23:14-22. [PubMed: 19290686]

Norris J, Stoner SA, Hessler DA, Zawacki T, George WH, Morrison DM, Davis KC. Cognitive mediation of alcohol's effects on women's in-the-moment sexual decision making. Health Psychology. 2009; 28:20-28. [PubMed: 19210014]

Otto, JM.; Davis, KC.; George, WH.; Heiman, JR. The influence of instructional set and cognitive strategies in the control of female sexual arousal. Poster session presented at the meeting of the Society for the Scientific Study of Sexuality, Puerto Vallarta; 2009, November; Puerto Vallarta, Mexico.

Paulhus, DL. Measurement and control of response bias. In: Robinson, JP.; Shaver, P.; Wrightsman, LS., editors. Measures of personality and social psychological attitudes. San Diego: Academic Press; p. 17-59.

Pfaus JG, Wilkins MF, Dipietro N, Benibgui M, Toledano R, Rowe A, Couch MC. Inhibitory and disinhibitory effects of psychomotor stimulants and depressants on the sexual behavior of male and female rats. Hormones and Behavior. 2010; 58:163-176. [PubMed: 19837072]

Prause N, Staley C, Finn P. Effects of acute ethanol consumption on sexual arousal and sexual risk taking. Archives of Sexual Behavior. 2011; 40:373-384. [PubMed: 21318417] 
Reynolds CF, Frank E, Thase ME. Assessment of sexual function in depressed, impotent, and healthy men: Factor analysis of a Brief Sexual Function Questionnaire for Men. Psychiatry Research. 1988; 24:231-250. [PubMed: 3406241]

Schacht RL, George WH, Heiman JR, Davis KC, Norris J, Stoner SA, Kajumulo KF. Effects of alcohol intoxication and instructional set on women's sexual arousal vary based on sexual abuse history. Archives of Sexual Behavior. 2007; 36:655-665. [PubMed: 17364230]

Schacht RL, George WH, Davis KC, Heiman JR, Norris J, Stoner SA, Kajumulo KF. Sexual abuse history, alcohol intoxication, and women's sexual risk behavior. Archives of Sexual Behavior. 2010; 39:898-906. [PubMed: 19728070]

Schacht RL, Stoner SA, George WH, Norris J. Idiographically determined versus standard absorption periods in alcohol administration studies. Alcoholism - Clinical and Experimental Research. 2010; 34:925-927.

Sobczak JA. Alcohol use and sexual function in women: A literature review. Journal of Addictions Nursing. 2009; 20:71-85.

Strassberg DS, Lowe K. Volunteer bias in sexuality research. Archives of Sexual Behavior. 1995; 24:369-382. [PubMed: 7661653]

Wilson GT, Lawson DM. Effects of alcohol on sexual arousal in women. Journal of Abnormal Psychology. 1976; 85:489-497. [PubMed: 965577]

Wilson GT, Lawson DM. Expectancies, alcohol, and sexual arousal in women. Journal of Abnormal Psychology. 1978; 87:358-367. [PubMed: 681606] 


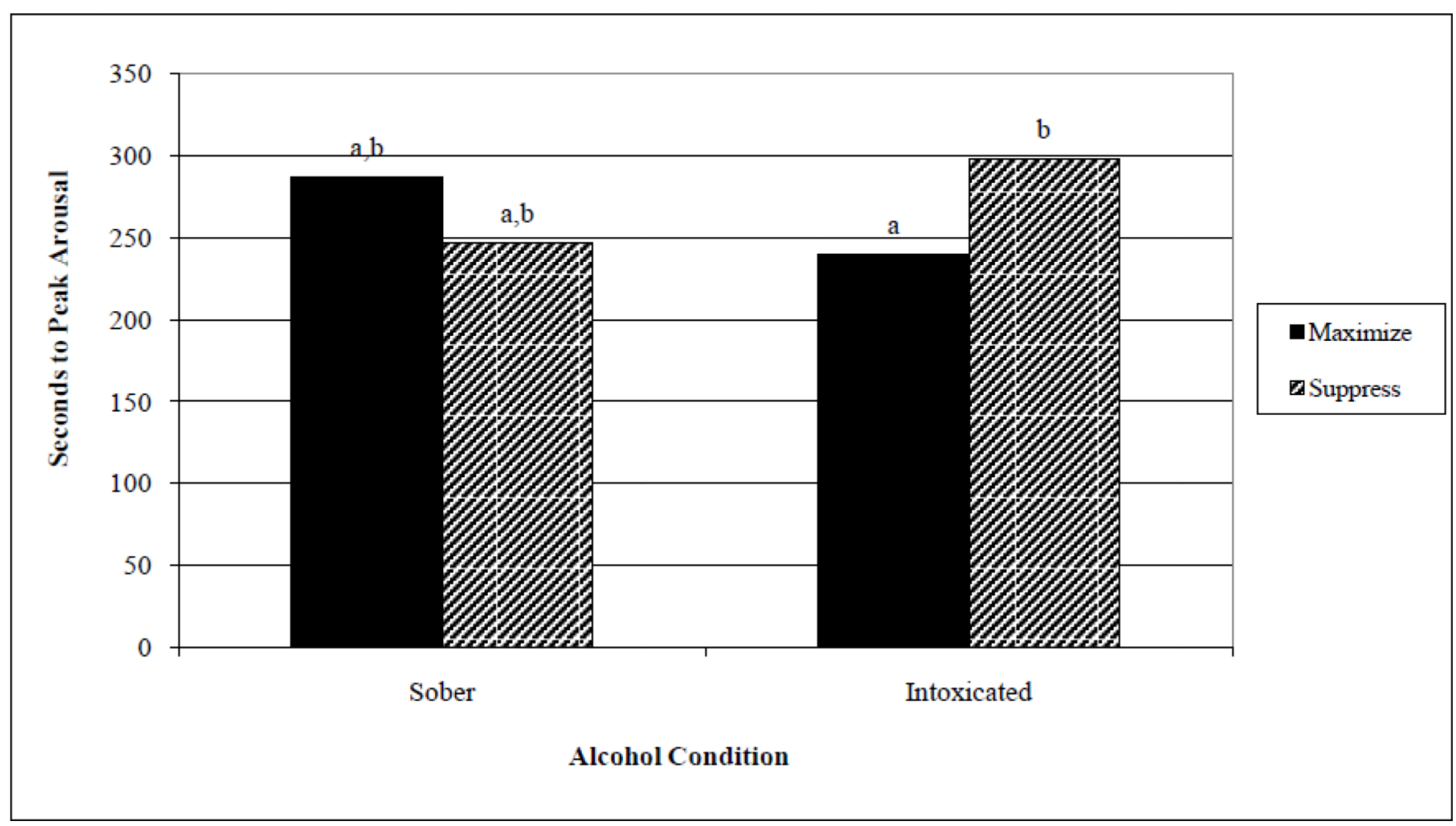

Fig. 1. 


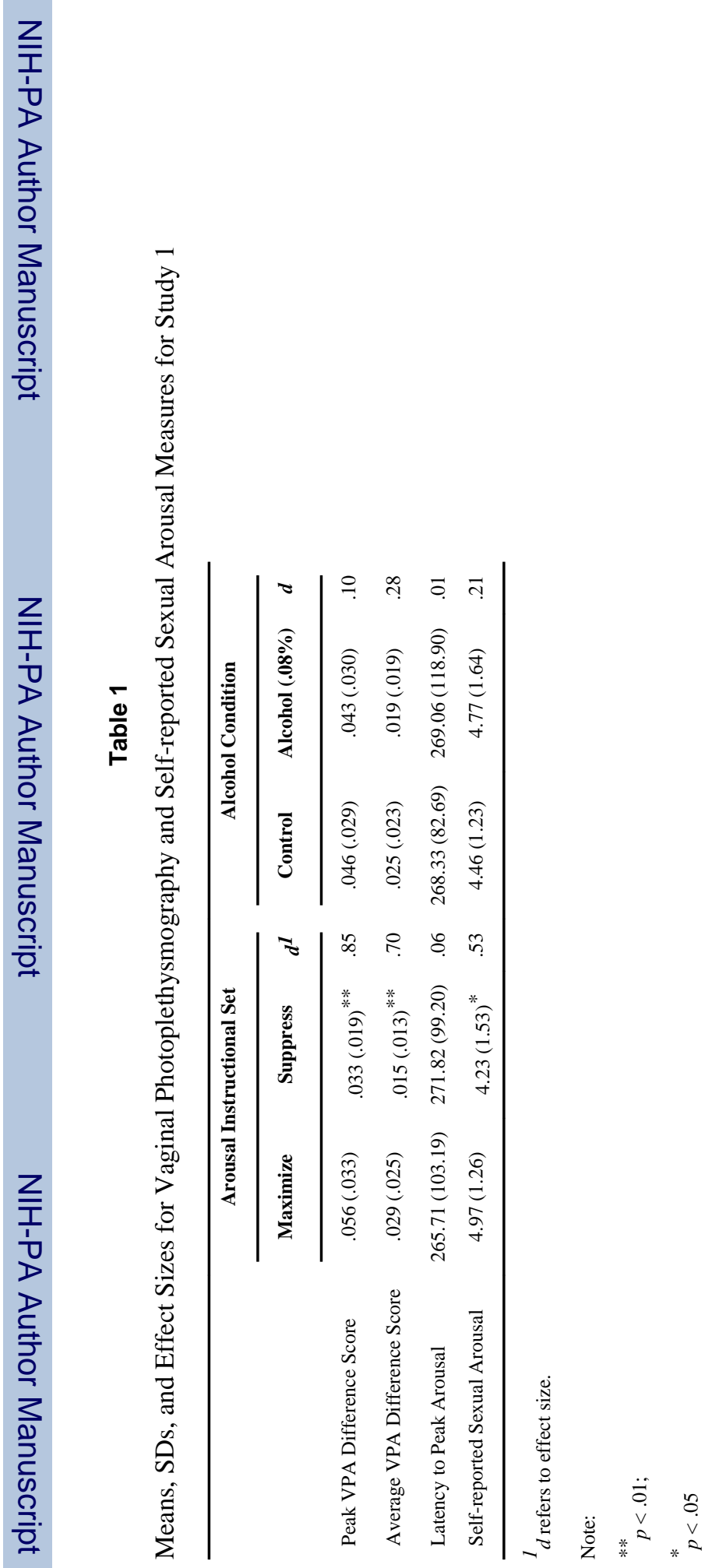




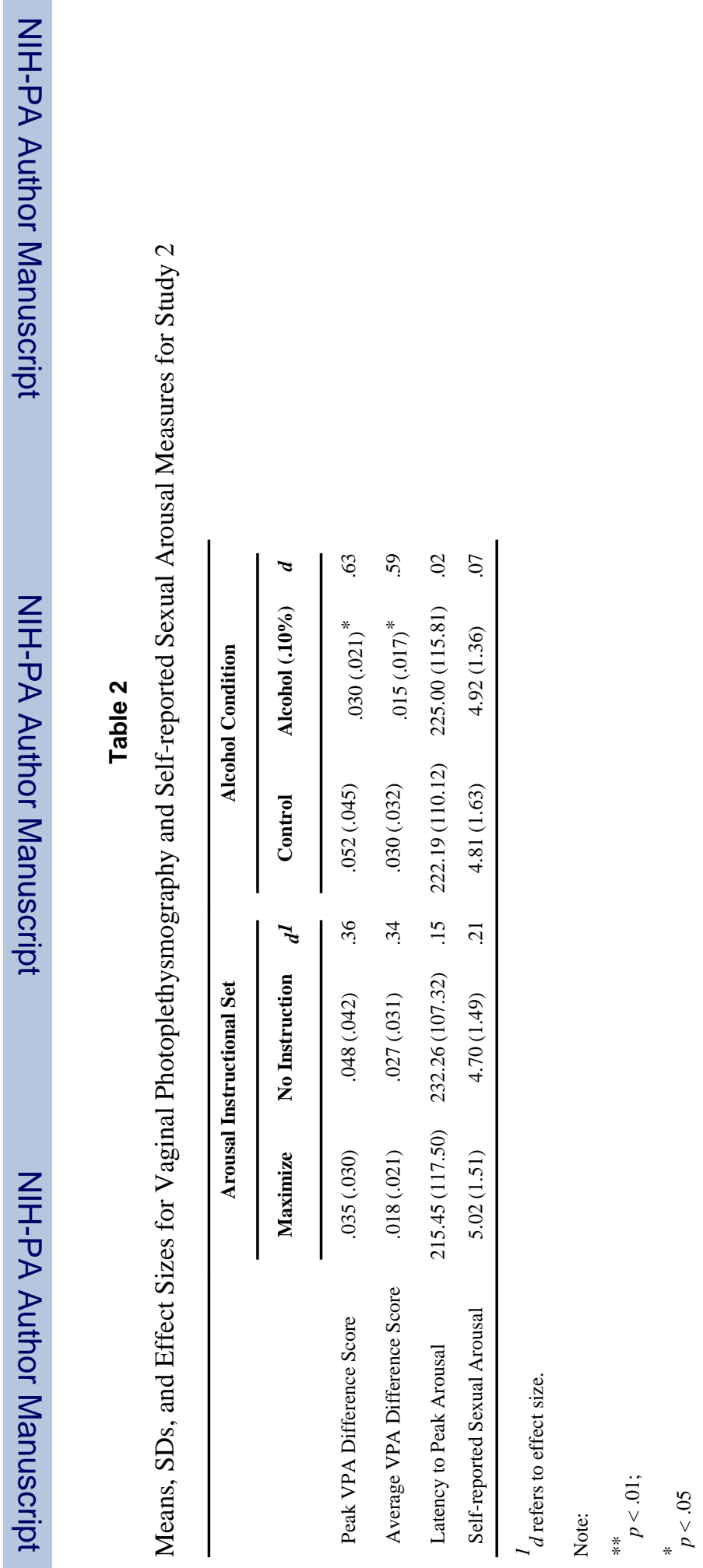

